\title{
Computational modelling of void growth in Phenolic Molding Compounds filled PolyPropylene from optical measurements
}

\author{
A.S. Caro ${ }^{\star}$, F. Bernardeau, D. Perrin, R. Leger, J.C. Benezet, P. lenny, \\ C2MA, IMT Mines Ales, Univ Montpellier, Ales, France \\ ${ }^{*}$ Corresponding authors: \\ anne-sophie.caro@mines-ales.fr
}

This paper has been first published online in Polymer Testing in September 10, 2018 (https://doi.org/10.1016/i.polymertesting.2018.09.008).

\section{Abstract}

Phenolic molding compounds (PMC), also known as Bakelite ${ }^{\mathrm{TM}}$, are the first synthetic plastics ever produced. They are still in use today in electrical, household and automotive applications. However, one major drawback of PMC is their lack of recycling option. Indeed, landfilling is still the main route of disposal, which constitutes a serious environmental burden. A new alternative recycling method is the use of comminuted PMC as filler in a thermoplastic matrix, in order to improve its mechanical and thermal properties. Several key parameters of the manufactured composite structure are the fillers size distributions, their loading percentage, and the adhesion at the filler/matrix interface. These parameters are related to mechanical properties of the composites, such as tensile strength. In this work, a polypropylene matrix is filled with $20 \%$ by weight of comminuted PMC $\left(d_{50}=24 \mu \mathrm{m}\right)$ functionalized with $3 \%$ by weight of PP-g-MA to improve matrix/fillers compatibility. A FE model was developed from the mechanical behavior of each component. PP matrix and PMC fillers are individually characterized from tensile tests instrumented with photomechanics for the matrix characterization and their behaviors were modelled through a set of numerical parameters (elasto-visco-plasticity with a Gurson criterion behavior for the matrix and damage elasticity for the fillers). Numerical simulations at several strain rates were conducted on representative volume element with various microstructures. Comparison between the determinist model results and the experimental data (strength, volumetric variation) shows that this type of modelling could be a predictive tool in order to design particulate composites with optimized 
mechanical properties. Hence, it is an adequate way to understand micromechanisms of deformation (damage, cavitation).

Keywords: Phenolic molding compounds, Damage, Volume change, Finite element

\section{Introduction}

As most thermoset materials, phenolic molding compound (PMC) wastes generate environmental problems and very few recycling solutions have been proposed for this type of material. In a previous paper, authors consider the possibility to use PMC as fillers in a polymer matrix [1], [2]. Mineral fillers such as calcium carbonate, talc, silica, clay, etc., are usually used to reduce costs, to give some flame-retardancy and/or in some cases to enhance mechanical material properties. The point of this article is to explore the mechanical enhancement possibilities of PMC incorporated into PolyPropylene (PP) matrix. From authors' knowledge, there are very few studies about its incorporation as a fillers into polymers [3], [4]. The major point is the weak interaction between PMC and PP (due to their chemical discrepancies) leading to poor mechanical properties. PP/PMC composites were compatibilized with maleic anhydride-grafted PP (PP-g-MA) to solve this drawback [5].

The present study aims at improving the comprehension of the micromechanics of deformation involved during a tensile test, especially the inelastic volume variation. To better understand the key role of the fillers on the material response, a predictive modeling is constructed from mechanical tests performed for each of composite phase components. The Gurson-Tvergaard-Needleman (GTN) model ([6]-[8]) has been used to describe the elasto-visco-plastic and damageable behavior of PP matrix. Actually, in references [9]-[11], authors used the same strategy to describe volume change in polyamide 11. A Kachanov elastic damage model is used to characterize the brittle behavior of bakelite [12]. Knowing the microstructure, a predictive Finite Element Analysis (FEA) was conducted as an useful tool to understand local phenomena leading to composite breakage.

The paper is structured as follows: section 2 presents materials and experiments; section 3 highlights modelling; section 4 discusses about results and section 5 brings a conclusion. 


\section{Materials and experiments}

\subsection{Materials}

\subsubsection{Bakelite}

Phenolic Molding Compounds known as Bakelite ${ }^{\mathrm{TM}}$ used in this study was recovered from WEEE (Waste Electrical and Electronic Equipment) casing. This resin is based on phenol and formaldehyde condensation. They were identified via Fourier Transform Infrared Spectroscopy (FTIR) analysis and manually sorted from other plastic parts. It was found in a previous research that this resin was reinforced with various fillers and additives such as calcium carbonate, silica, magnesium carbonate and wood. Bakelite densities is between 1.31 and $1.39 \mathrm{~g} / \mathrm{L}$ [2].

PMC waste is therefore transformed into usable fillers of appropriate size by comminution. The polydispersity of PMC filler size distribution was quantified by laser diffraction Particle Sizing Technique (PSD) (Figure 1). The procedure is fully described in [2].

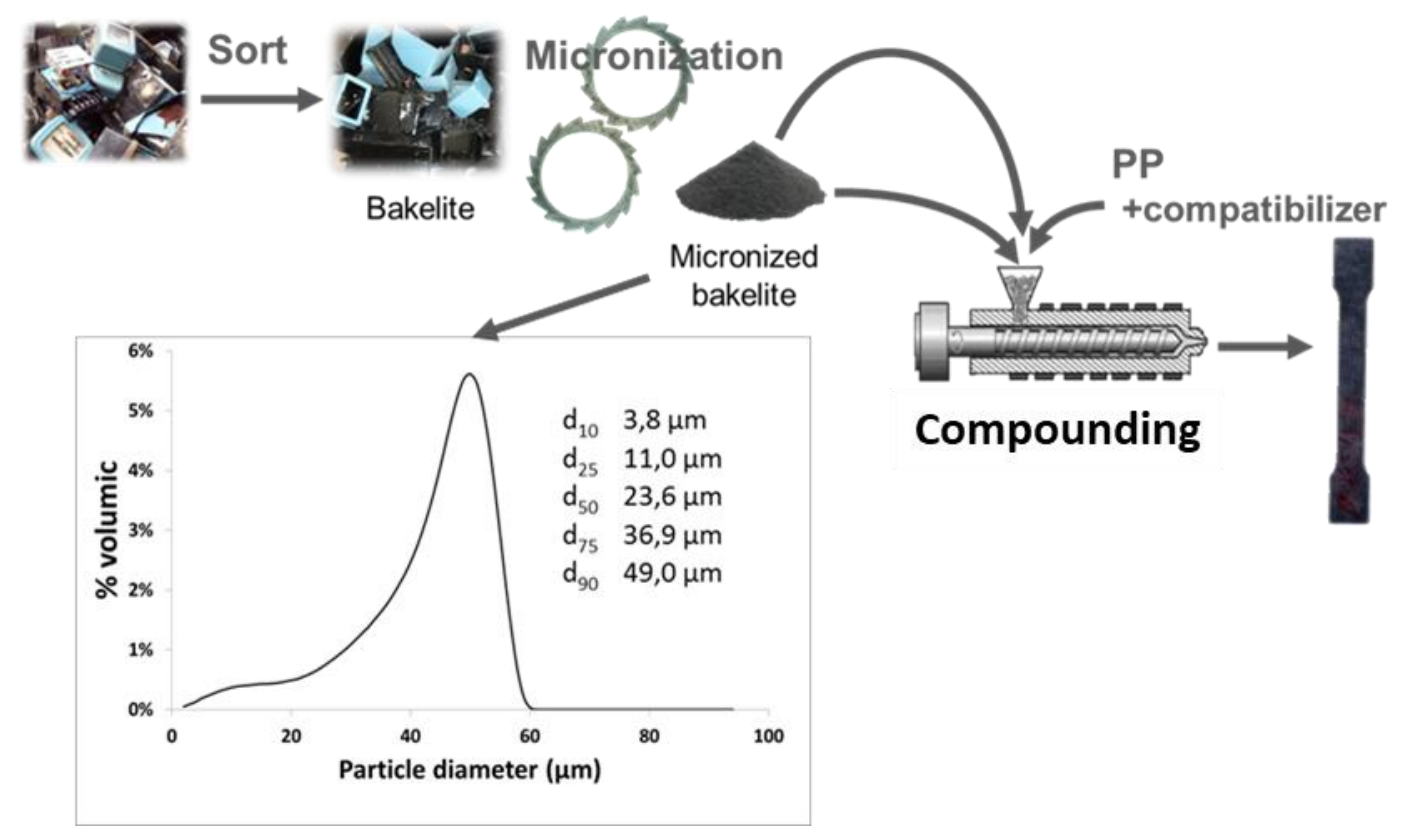

Figure 1: Recycling of Bakelite, from WEEE to its incorporation in PP matrix with a controlled size distribution 
A Polypropylene 576P was kindly provided by Sabic. It is a PP homopolymer with narrow molecular weight distribution and a MFI of $19\left(230^{\circ} \mathrm{C}, 2.16 \mathrm{~kg}\right)$. A Polypropylene grafted with maleic anhydride (PP-g-MA) Orevac CA100 from Arkema was also used in this study as compatibiliser.

\subsubsection{Composite}

Particulates composite were prepared by extrusion. All materials were dried at $80^{\circ} \mathrm{C}$ during $24 \mathrm{~h}$ in an air circulating oven prior to compounding. A Clextral BC21 corotating twin screw extruder was used. The temperature was set to $220^{\circ} \mathrm{C}$ at the primary feeding port, and to $210^{\circ} \mathrm{C}$ at the extrusion head. A rotation speed of 250 rpm was used. An overall extrusion rate of $5 \mathrm{~kg} / \mathrm{h}$ was set for all composite. PMC fillers were fed in a port located in the middle of the barrel. PP-g-MA was dry-mixed with polypropylene before processing. The extruded material was cooled in a water bath and granulated. Compounds with $20 \%$ in weight of fillers were produced.

After compounding, extruded materials were dried at $80^{\circ} \mathrm{C}$ during $24 \mathrm{~h}$ in an air circulating oven. The pellets were then injection molded with a Krauss Maffei KM50T180CX. Dog bone samples (ISO 1A according to ISO 527-2) were injected for mechanical testing.

The crystallinity was measured by differential scanning calorimetry (DSC). For pure polypropylene, a crystallinity of $48 \%$ was measured. The crystallinity of composites is around $46 \%$.

In order to improve the adhesion between the filler and the matrix, $3 \%$ by weight of PP-g-MA was added into the composite.

Three different formulations (PP, PP with PMC with or without PP-g-MA), presented in Table 1, were thus prepared for this study. In the following, PP, PP/PMC, PP/PMC/gMA denote respectively the neat PP, PP with 20\% in weight of Bakelite ${ }^{\mathrm{TM}}$ and PP with $20 \%$ in weight of Bakelite ${ }^{\mathrm{TM}}$ and $3 \%$ by weight of PP-g-MA.

Table 1: Materials under study

\begin{tabular}{lccc}
\hline Acronym & PP & 20\%w PMC & $3 \% w$ PP-g-MA \\
\hline PP & $\times$ & & \\
\hline
\end{tabular}




\begin{tabular}{llll}
\hline PP/PMC & $\times$ & $\times$ & \\
PP/PMC/gMA & $\times$ & $\times$ & $\times$ \\
\hline
\end{tabular}

\subsection{Instrumented mechanical tests}

\subsubsection{Principle}

Uniaxial tensile tests were conducted on a Zwick TH010 universal testing machine according to the ISO 527 standard. The crosshead speed is equal to 5 and 50 $\mathrm{mm} / \mathrm{min}$ (corresponding respectively to strain rates $8.10^{-4} / \mathrm{s}, 8.10^{-3} / \mathrm{s}$ ) as we suspected a time dependence behavior for the PP matrix. The tensile test is conducted up to the rupture of the specimen. The software used is TestXpert $\circledast$ and allows the recording of time, load and elongation. Let's define by $\boldsymbol{x}$ the tensile direction, $\boldsymbol{y}$ perpendicular to $\boldsymbol{x}$ and in the planar surface and $\boldsymbol{z}$ the out of plane direction.

The nominal stress is given by the following expression (eq. 1):

$$
\sigma=\frac{F}{S_{0}}
$$

where $F$ is the recorded load and $S_{0}$ the initial sample section surface.

The optical extensometer involves a high resolution Charge-Coupled Device (CCD) camera (Redlake Megaplus II, $1920 \times 1080$ contiguous and square pixels, coded in 256 grey levels), set in front of the specimen, which records images during the test (Figure 2). The optical axis of the camera remains perpendicular to the in-plane surface of the specimen during the test. The images acquisition is commanded by a LabVIEW $^{\circledR}$ software which allows the simultaneous acquisition of the images and the data from the testing machine (such as load and crosshead displacement). According to the test speed used, images are recorded every 2 s. The scale factor is fixed to 42 $\mu \mathrm{m}$ per pixel.

The in-plane strains $\varepsilon_{x}, \varepsilon_{y}$ were deduced from digital image correlation (DIC) following a method well described in [13]. A transverse isotropic assumption is considered and was validated in a previous study on polycarbonate nanocomposite specimen submitted to uniaxial tensile test [13]. 
The volumetric strain is defined as (eq. 2):

$$
\frac{\Delta V}{V_{0}}=\operatorname{tr}(\varepsilon)=2 \varepsilon_{y}+\varepsilon_{x}
$$

From (eq. 2) the part of elastic volumetric strain can be computed (eq. 3):

$$
\left(\frac{\Delta V}{V_{0}}\right)_{\text {elas }}=\frac{\sigma}{E}(1-2 v)
$$

where $E$ and $v$ are the elastic material parameters (Young modulus and Poisson's ratio).
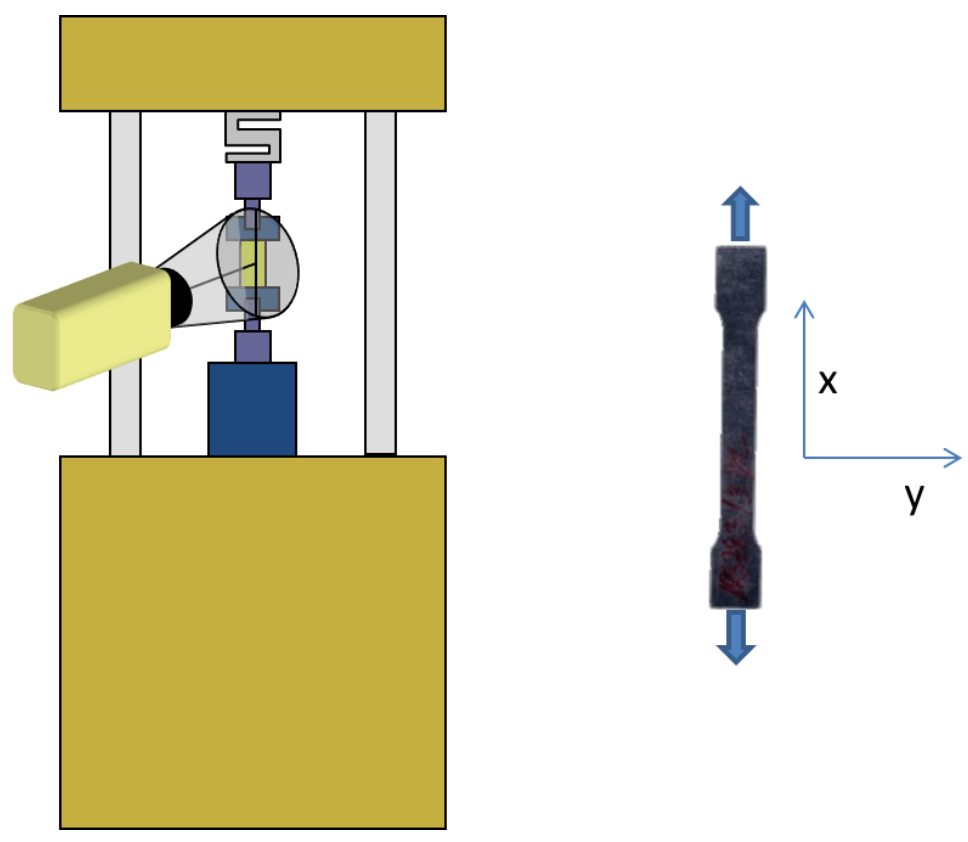

Figure 2: Testing apparatus

\subsubsection{Results}

\subsubsection{PP}

As a first result, the PP matrix behaves as a damageable viscoelastic material. Its global behavior is time dependent (Figure 3 (a)) and its volumetric strain (deduced from photomechanics and eq. 2) does not follow an elastic law (see for example the discrepancies between elastic and global volumetric strain for the lower imposed strain rate in Figure $3(b))$. 


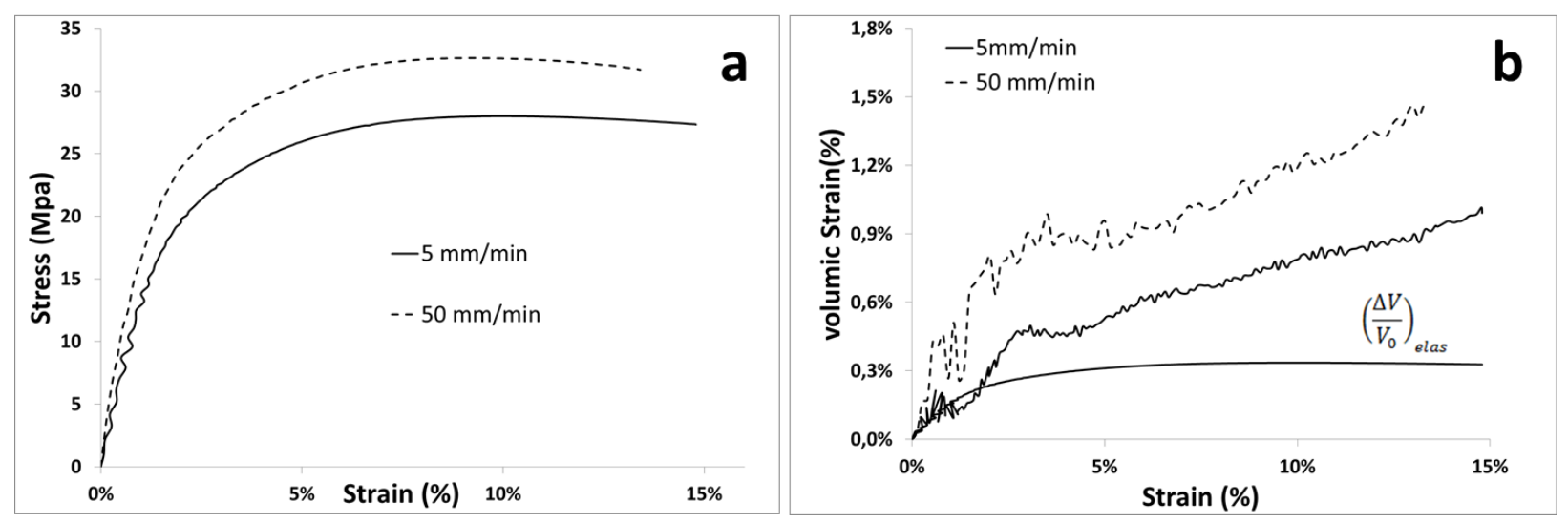

Figure 3: Stress vs. strain (a), Volumetric Strain (b) in uniaxial tensile tests at several imposed strain rates for PP matrix

\subsubsection{Bakelite $^{\mathrm{TM}}$}

The mechanical properties of Bakelite ${ }^{\mathrm{TM}}$ were analyzed through 3-points flexural tests in Zwick TH010 equipment. A load was applied on the specimen at $2 \mathrm{~mm} \cdot \mathrm{min}^{-1}$ crosshead motion rate. Eight specimens were analyzed with dimensions mentioned in Figure 4. The material showed an elastic-brittle behavior. Average flexural modulus (7.8 GPa), strength (85.8 MPa) and ultimate strain $(1.1 \%)$ are therefore deduced.
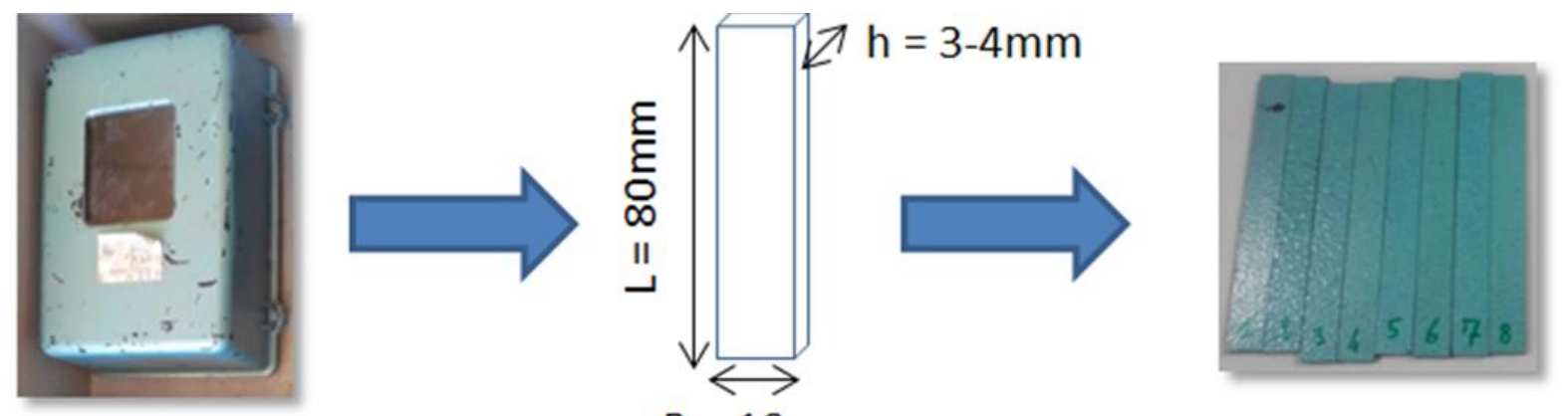

$$
\mathrm{B}=10 \mathrm{~mm}
$$

Figure 4: Bakelite ${ }^{\mathrm{TM}}$ samples for mechanical testing

\subsubsection{Composites}

Once fillers are added to PP matrix and possibly with PP-g-MA, the global shape of the stress/strain curve is unchanged. As expected, the Young's modulus is increased by the incorporation of PMC (independently of the presence of PP-g-MA) just like the associated maximum stress (for the sample with PP-g-MA). The Poisson ratio remains unchanged (see Table 2). The ultimate strain is lowered by the incorporation of fillers since they act as defects which initiate the composite breakage. The 
presence of PP-g-MA produces the opposite effect (see Figure 5 (a) for a lower imposed speed). The measured volumetric strain is higher for the composites in comparison of the neat polymer (Figure 6 (b)). For example, at $5 \%$ of strain, volumetric strain value is 5 times (respectively 2 times) higher for the composite with PMC (with both PMC and PP-g-MA). The fillers increase the composite volumetric variation. The presence of PP-g-MA into the composite reduces this variation. The anhydride maleic could maintain cohesion at the interface matrix/fillers and limits the global volumetric variation. This latest assumption will be checked with an in-situ tensile test with ESEM equipment (see the next part 2.3: microscopic observations).

\begin{tabular}{ll|l|l}
\hline & PP & PP/PMC & PP/PMC/gMA \\
\hline E (MPa) & 1701 & 2197 & 2839 \\
$v$ & 0.38 & 0.38 & 0.38 \\
$\sigma_{u}(\mathrm{MPa})$ & 25 & 22 & 30 \\
$\varepsilon_{u}(\%)$ & 0.15 & 0.06 & 0.1 \\
\hline
\end{tabular}

Table 2: Elastic and ultimate properties deduced from experiments for PP, PP/PMC and PP/PMc/gMA composites

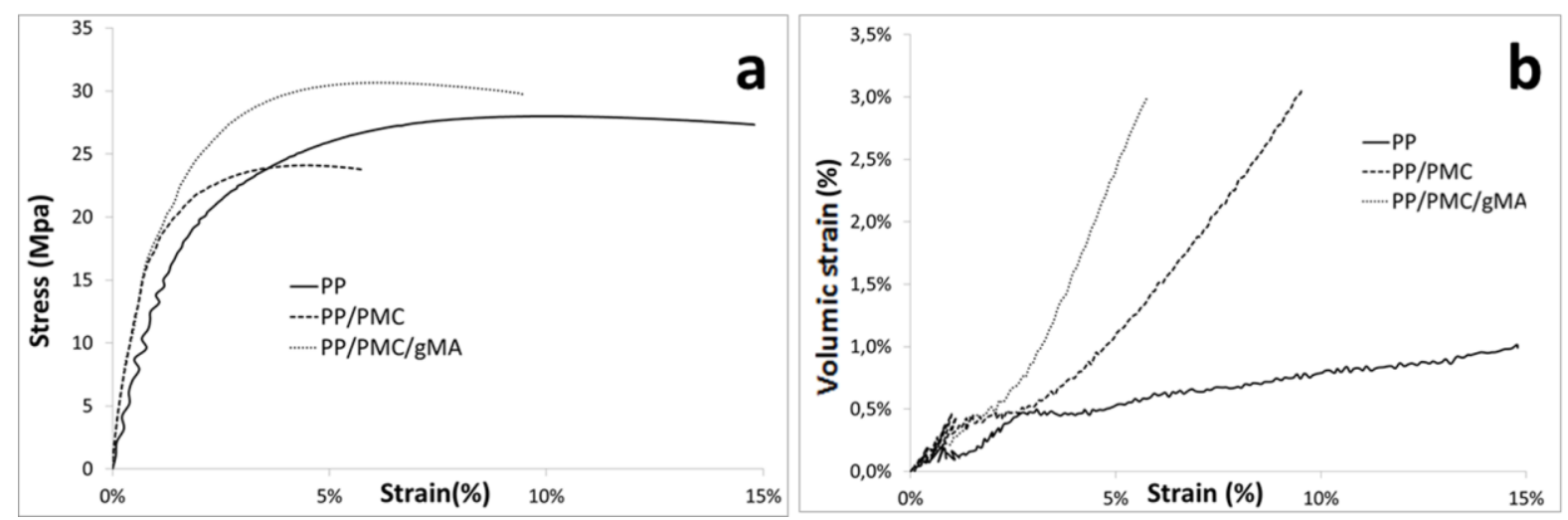

Figure 5: Stress vs. strain (a), Volumetric Strain (b) in uniaxial tensile tests at 5 $\mathrm{mm} / \mathrm{min}$ for PP matrix and composites with and without PP-gMA

\subsection{Microscopic observations}




\subsubsection{Post mortem observations}

Tensile test specimens were cryo-fractured and the central zone of the samples (perpendicular to the flow direction) was observed using a Quanta SEM instrument (FEl, USA) under an accelerating voltage of $12.5 \mathrm{kV}$. As a first observation, the global dispersion into PP/PMC and PP/PMC/g-MA composites is similar (Figure 6 (a) and (b)). Besides, there is no visible adhesion between fillers and PP matrix due their opposite polarity (Figure 6 (c)). The PP-g-MA improves the filler/matrix compatibility by generation of observable links (Figure $6(d)$ ).
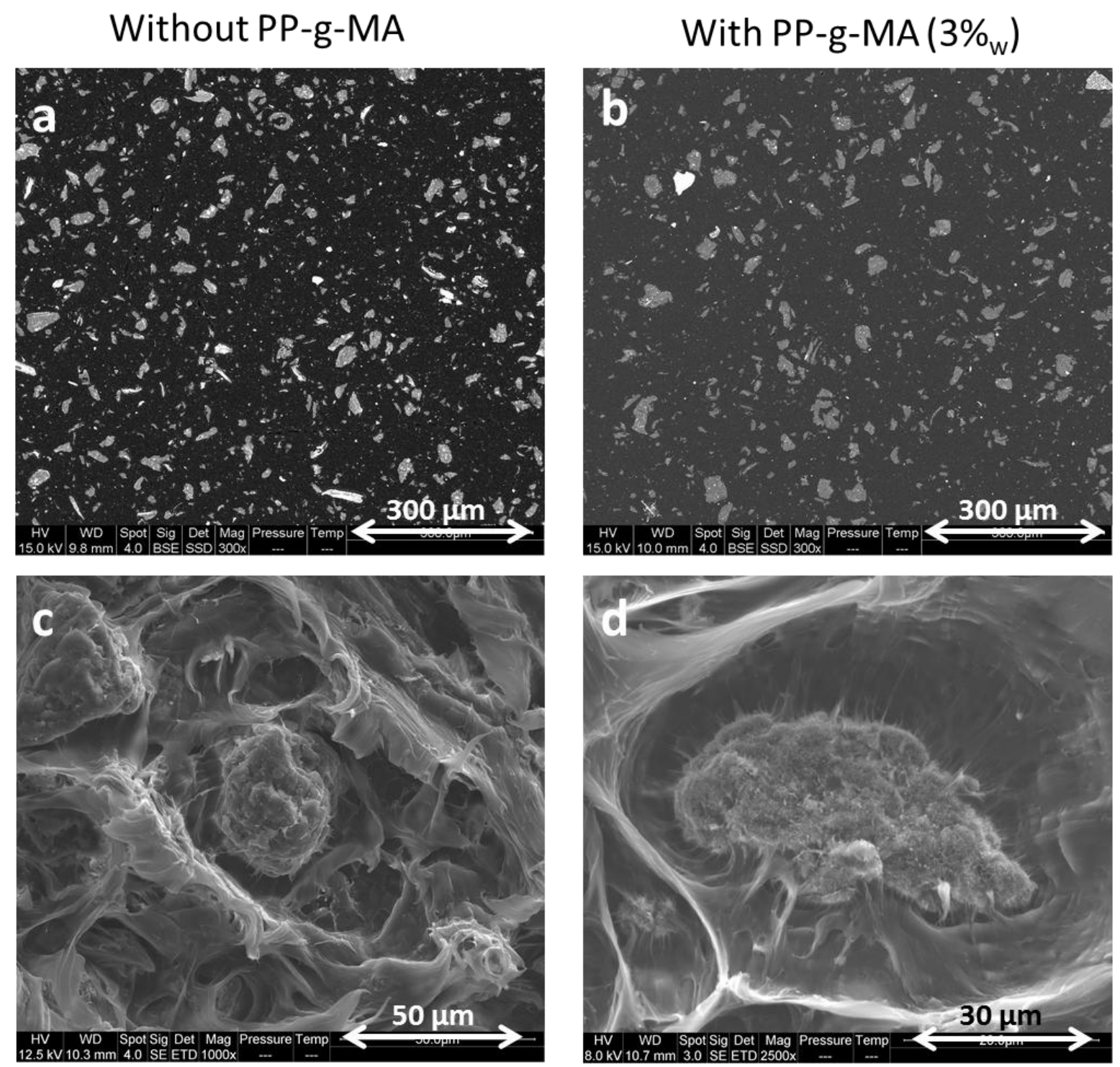

Figure 6: ESEM micrographs of PP with $20 \%$ of PMC, scale factors 150 (a), 250 (b), 1000 (c), 2500 (d) 
Tensile tests were carried out by ESEM Quanta FEG 200 on rectangular shaped samples $\left(50 * 10 * 4 \mathrm{~mm}^{3}\right)$ with a $10 \mathrm{~mm}$ gauge length and repeated three times. Two notches were performed symmetrically on each side of samples (corresponding to a minimal section of $6 * 4 \mathrm{~mm}^{2}$ ) to initiate the fracture in the observation field (Figure 7). The device is equipped with a $5 \mathrm{kN}$ load cell and the test speed is set at $5 \mathrm{~mm} / \mathrm{min}$. One image is recorded every ten seconds and force and displacement are recorded at a frequency of $2 \mathrm{~Hz}$.

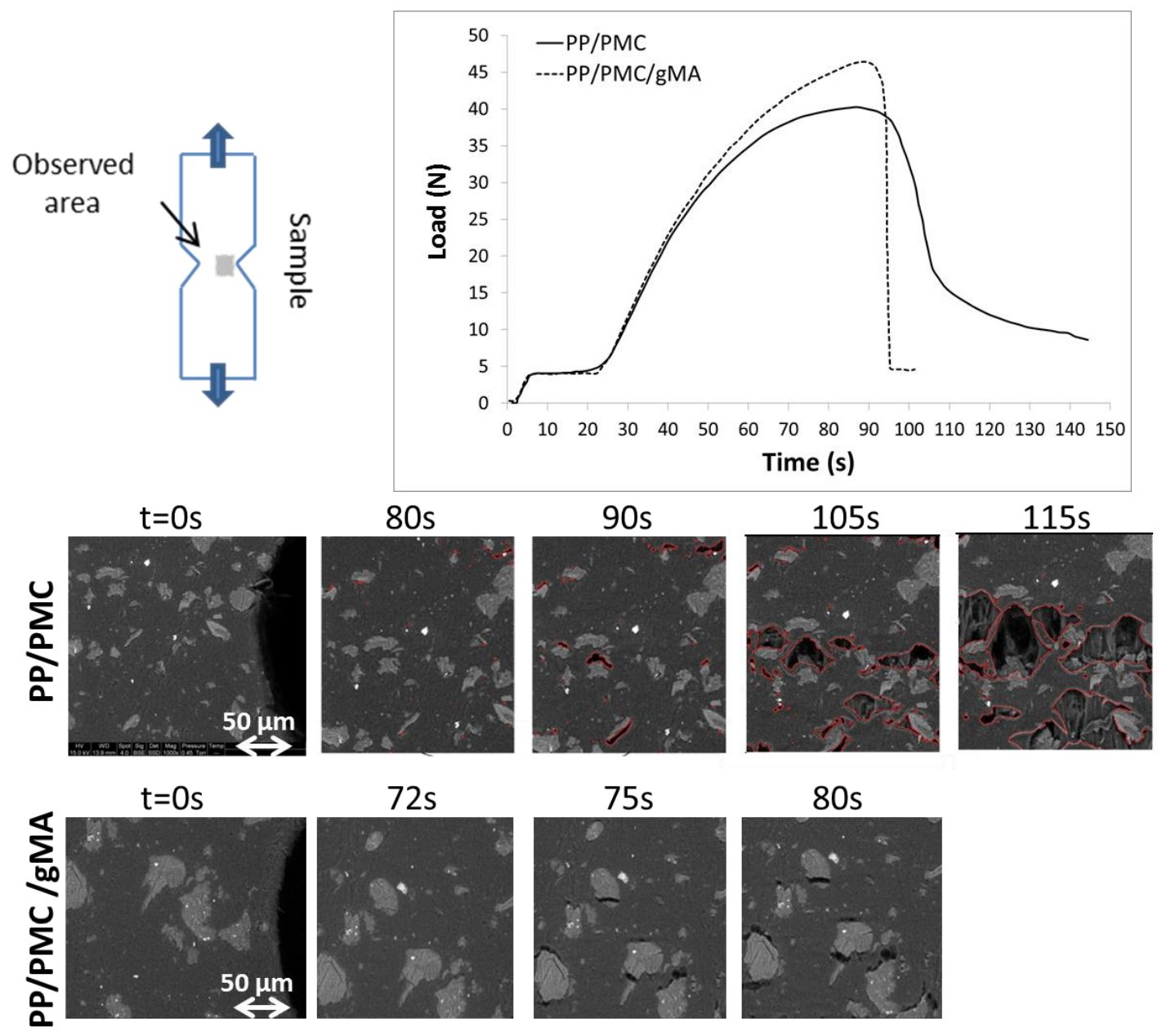

Figure 7: Tensile tests on PP with 20 wt\% of PMC (with and without PP-g-MA), ESEM observation near to the notch

At the beginning of the test, a first stage, corresponding to the equipment misalignment, is observed until 25s. After that, an elastic stage occurs for which no structural variation is observed. For the PP/PMC composite, structural modifications 
such as decohesion at the interfaces clearly appear around the apparition of the maximum stress. At the same level of stress, the interface for the PP/PMC/gMA composite seems to be stronger, since the previous observed decohesion is replaced by a breakage inside the Bakelite $^{\mathrm{TM}}$ (Figure 7).

\section{Modelling}

\subsection{Behavior}

The literature shows an important number of constitutive models, namely "damaged model", able to account mechanical degradation in ductile materials. Usually, these models include new internal variables and evolution laws coupled to plasticity problems. Relevant approaches are, for example, the works of Gurson [6], Lemaitre [14] and Rousselier [15]. The present work focuses on Gurson-type (for PP) and Lemaitre-type (for Bakelite ${ }^{\mathrm{TM}}$ ) material models.

\subsubsection{PP: modeling from a Gurson-type damage model}

Gurson-type approach is based on mechanics of porous media. The damage indicator, related to the degradation of the material, is the void volume fraction (i.e. the porosity) which progressively down-scale the yield surface. In the early eighties Tvergaard and Needleman [16], [17] extended the Gurson approach, namely GNT model, including material hardening, multiple voids and void coalescence. More recently, this model has been written within the thermodynamical framework of Lemaitre and Chaboche [18].

The volume strain, usually used to quantify the porosity, is measured by some authors with SEM, wide-angle X-ray scattering (WAXS) or small-angle X-ray scattering (SAXS) [9], [19]-[22]. Even if initial porosity has not been observed for the neat PP, this approach remains consistent as observed volume change is simultaneous to void nucleation and growth (see results of section 2.3).

In this damage formulation the yield criterion uses hydrostatic pressure and porosity: the criterion of plasticity and the plastic potential, depend on a macroscopic stress $\sigma_{*}$ and an effective porosity fraction $f^{*}$ in the following way (eq. 4): 


$$
\frac{3 J_{2}(\tilde{\sigma})}{\sigma_{*}^{2}}+2 f^{*} q_{1} \cosh \left(\frac{q_{2} I_{1}(\tilde{\sigma})}{2 \sigma_{*}}\right)-\left(1+q_{1}^{2} f^{* 2}\right)=0
$$

where $\tilde{\sigma}$ is the stress tensor, $J_{2}=\frac{1}{2} \operatorname{dev}(\tilde{\sigma}): \operatorname{dev}(\tilde{\sigma}), I_{1}=\operatorname{trace}(\tilde{\sigma})$ and $q_{1}, q_{2}$ are model parameters.

Effective stress $\sigma_{*}$ is implicitly evaluated from eq. 4.

The plastic flow potential is written as (eq. 5):

$$
f=\sigma_{*}-R(p), \quad R=R_{0}+Q_{1}\left(1-e^{-b_{1} p}\right)
$$

where $p$ is the effective plastic strain and $R_{0}, Q_{1}, b_{1}$ are material parameters.

The viscoplastic strain is written with a Norton law:

$$
\dot{\tilde{\varepsilon}}=(1-p) \dot{p} \tilde{n}_{*}, \quad \tilde{n}_{*}=\frac{\partial \sigma_{*}}{\partial \tilde{\sigma}}, \quad \dot{p}=\left(\frac{f}{K}\right)^{n}
$$

where $n, K$ are material parameters.

The volumetric change can therefore be divided in two parts:

$$
\frac{\Delta V}{V_{0}}=\left(\frac{\Delta V}{V_{0}}\right)_{\text {elas }}+\left(\frac{\Delta V}{V_{0}}\right)_{\text {cavitation }}
$$

\subsubsection{Bakelite: modeling from continuum damage mechanics}

Lemaitre-type approach is based on continuum damage mechanics. A macroscopic damage variable $d$ is introduced to describe the progressive degradation of the material [12]. In Bakelite ${ }^{\mathrm{TM}}$, we suppose that the damage accumulation occurs under elastic deformation as Bakelite ${ }^{\mathrm{TM}}$ is an elastic brittle material. Then the stress can be expressed as follows:

$$
\tilde{\sigma}=(1-d) \widetilde{D}_{\text {elas }}: \tilde{\varepsilon}_{\text {elas }}, d=\alpha\left[\max \sqrt{\frac{1}{2} \tilde{\varepsilon}_{\text {elas }}: \widetilde{D}_{\text {elas }}: \tilde{\varepsilon}_{\text {elas }}}-\sqrt{Y_{0}}\right]
$$

where $Y_{0}$ and $\alpha$ are material parameters and $\tilde{\varepsilon}_{\text {elas }}$ is the elastic part of the strain tensor. $\widetilde{D}_{\text {elas }}$ is the elasticity tensor depending on Young modulus and Poisson's ratio.

\section{Discussion}

The non-linear numerical simulation has been carried out using the FE software Zebulon, which has been developed at Mines Paristech [23]. 


\subsection{Identification of material parameters}

Material parameters were identified by using an inverse method of optimization of ZeBuLON software.

\subsubsection{PP matrix}

The method used a minimization algorithm based on that of Levenberg Marquardt leading to a set of parameters (given in Table 3) that conduct to a good accordance between simulated and experimental stress/strain (Figure 8 (a)). The porosity associated to cavitational volumetric strain is deduced from eq. 7 and compared to the experimental one in Figure 8 (b) for both imposed strain rates. Young's modulus and Poisson's ratio are deduced from optical measurements at low strain.

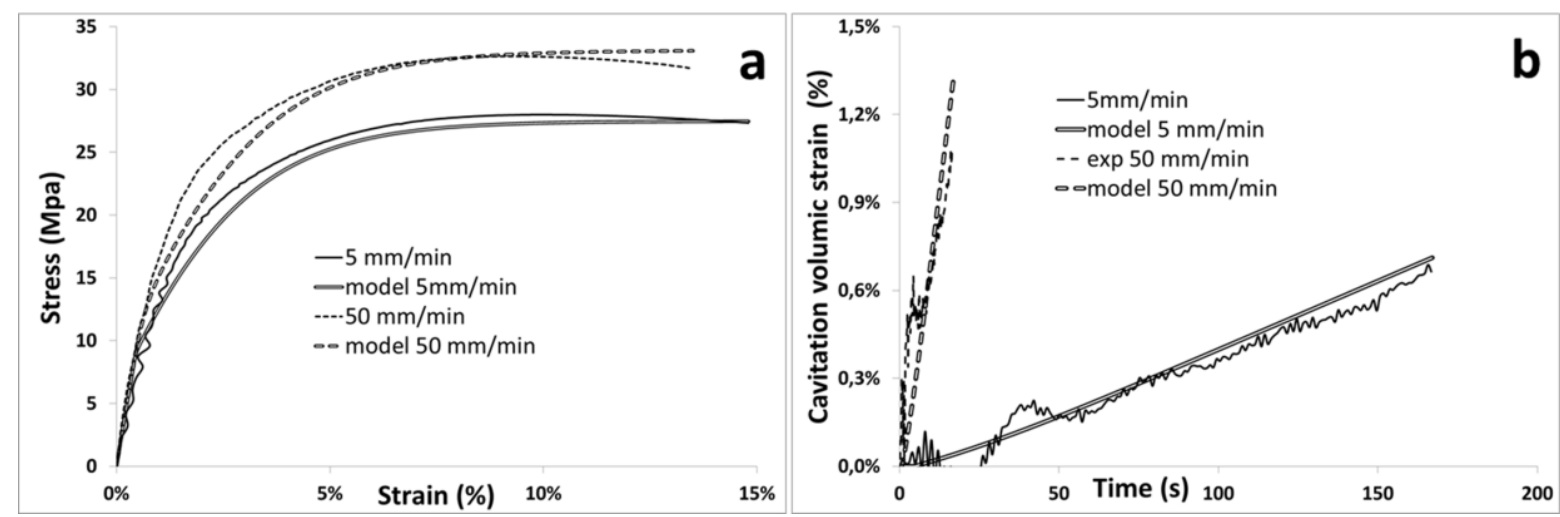

Figure 8: Modelling of PP at several imposed speed, stress vs. strain (a) cavitation volumetric strain vs. time (b)

\subsubsection{Bakelite $^{\mathrm{TM}}$ fillers}

As a first result, flexural tests were used for Bakelite ${ }^{T M}$ mechanical properties [2]. Bakelite mechanical properties are summarized in Table 2. Ultimate stress $\left(\sigma_{u}\right)$ and strain $\left(\varepsilon_{u}\right)$ are recorded in this table.

\begin{tabular}{ll|ll}
\hline & Bakelite $^{\text {TM }}$ & \multicolumn{1}{c}{ PP } \\
\hline E (MPa) & 7800 & E (MPa) & 1701 \\
$v$ & 0.38 & $v$ & 0.38
\end{tabular}




\begin{tabular}{ll|ll}
$\mathrm{Y}_{0}(\mathrm{MPa})$ & 0.1391 & $\mathrm{q}_{1}, \mathrm{q}_{2}(\mathrm{MPa})$ & $1 ; 1$ \\
$\alpha$ & 0.9 & n, K (MPa.s) & $1.21 ; 504.57$ \\
$\sigma_{u}(\mathrm{MPa})$ & 50 & $\mathrm{R}_{0}, \mathrm{Q}(\mathrm{MPa}), \mathrm{b}$ & $10 ; 21.6 ; 67.9$ \\
$\varepsilon_{u}(\%)$ & 2.5 & & \\
\hline
\end{tabular}

Table 3: Material parameters for PP and Bakelite ${ }^{\mathrm{TM}}$

\subsection{Predictive modelling}

For sake of simplicity, a plane stress assumption is assumed for the numerical computations. This strong hypothesis is very far from reality; nevertheless finite elements evaluations are faster in this case and a 2D microstructure enables, in a first approach, to understand micro mechanisms of deformation. The interface between matrix and fillers is assumed to be perfect and phase properties are described through the set of parameters of Table 3. As the PP crystallinity is not such impacted by the fillers (see section 2.3.1), we assume that the properties of PP matrix into the composite are similar to those of the neat PP.

An example of microstructure (generated via DIGIMAT software) is given in Figure 9, the granulometry of the fillers size is the one mentioned in Figure 1. Mesh microstructure based on triangular elements and boundary conditions are given in this table. We assume the structure symmetry (on the line of symmetries components of displacements are zero). A prescribed displacement $(10 \mathrm{~mm}$, corresponding to $10 \%$ of strain) is applied on the top of the sample. The size of this representative volume element (RVE), namely box length in Figure 9 and the number of elements correspond to minimal values leading to a convergent stress/strain numerical data. This RVE model is thus useful to predict the mechanical properties of PP/Bakelite composites. 

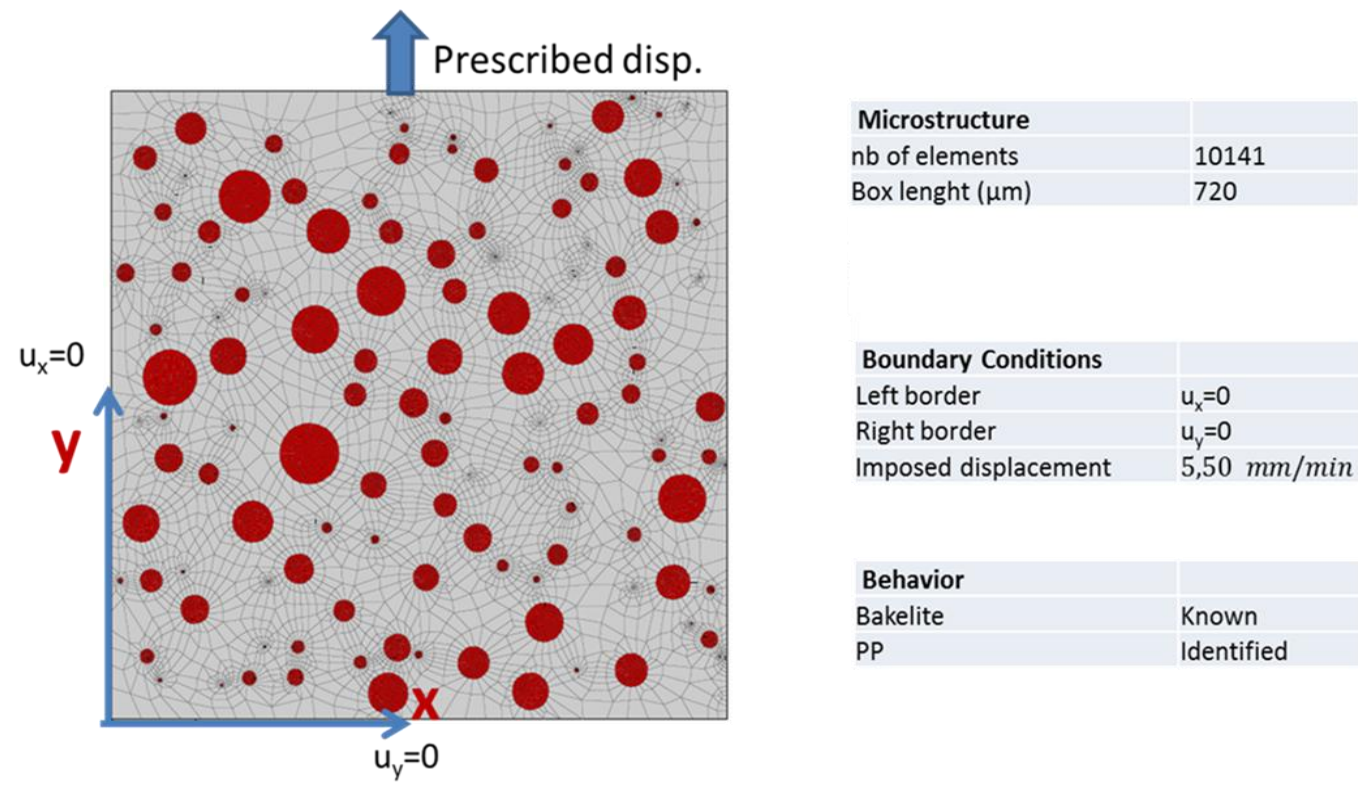

Figure 9: Finite Element Modelling: structure and boundary conditions

Numerical simulations were conducted at several speeds (5 and $50 \mathrm{~mm} / \mathrm{min}$ ). Comparison between numerical and experiments is given in Figure 10 (a) for the PP/PMC/g-MA for which the adhesion between matrix and fillers is improved. As a first result, input materials parameters lead to good predictive modelling for both speeds. As an example, stress cartography is added in the figure. Fillers reach a high level of stress in comparison with the PP matrix (red color in the figure). Regarding the volumetric strain, once again the prediction gives satisfactory results. Nevertheless a deviation is observed from $5 \%$ of strain: the model underestimates the volume change. This result is a consequence of material modelling assumption: the filler damage should be associated to an additional volumetric variation and to interfacial decohesion.
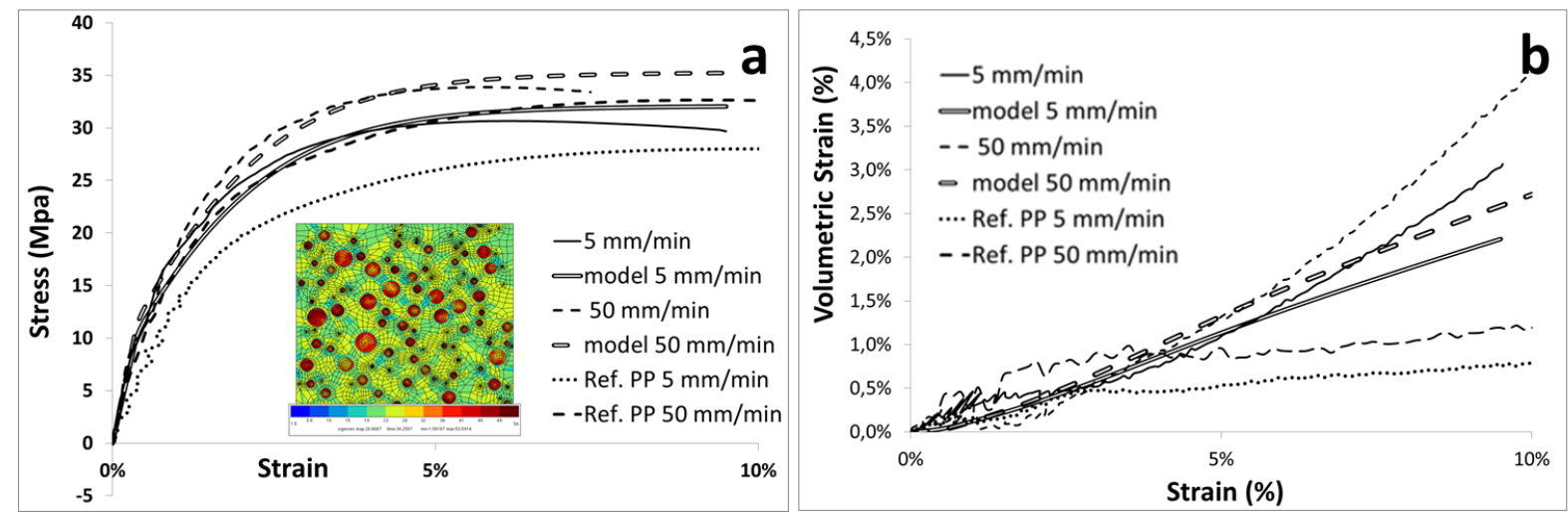
Figure 10: Stress vs. strain (a) volumetric strain vs. strain (b) for PP/PMC/gMA composite at several imposed speeds, experiments and modelling

The major advantage of numerical simulations is the access to microstructural information. For example, the average stresses in both matrix and fillers and the damage area in the Bakelite ${ }^{\mathrm{TM}}$ can be computed. This latest value corresponds to the relative surface of fillers that have reached breakage ( $d=1$ in eq. 8). Average stress for PP in PP/PMC/g-MA composite is plotted vs. strain in Figure 11 (a) for both imposed speeds. On average, the higher the imposed speed, the higher the average stress in the matrix, unlike bakelite with an average behavior almost insensitive to the applied speed. The fillers do not impact the matrix behavior from this point of view. Regarding the Bakelite ${ }^{\mathrm{TM}}$ (Figure 11 (b)) the initiation of damage occurs at the same time, independently of the imposed speed, whereas its development is faster as the velocity increases. Tests at high speeds are therefore suitable to discriminate interfacial properties in a composite as the particles involve a faster damage.

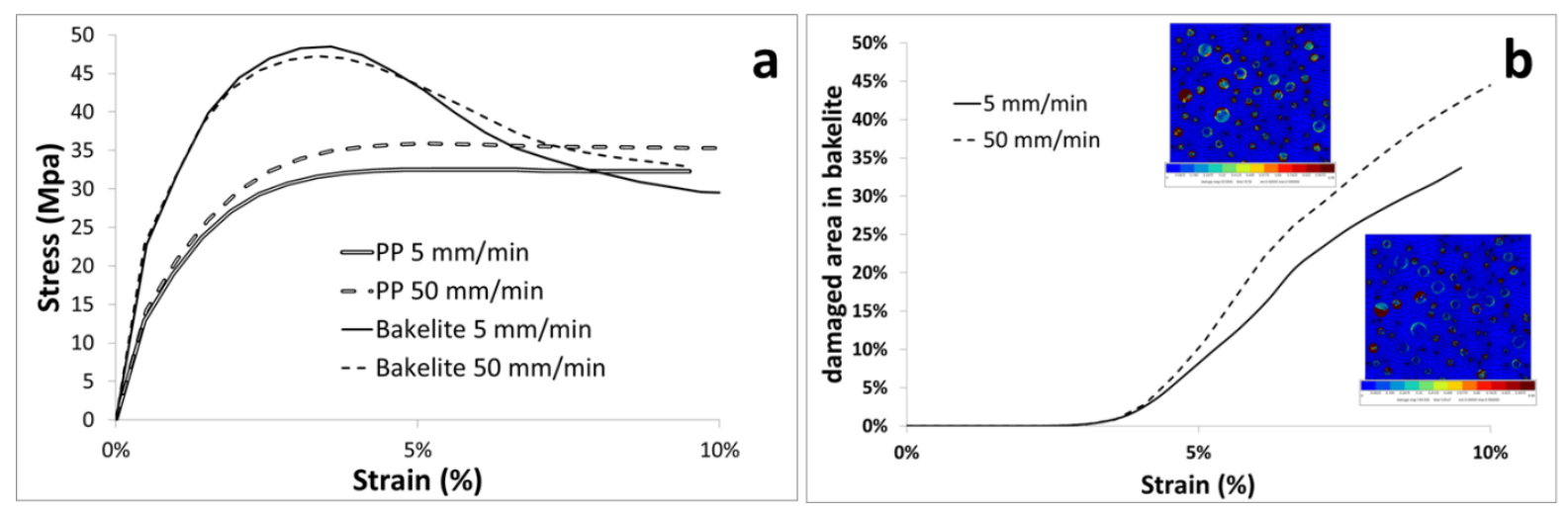

Figure 11: Average stresses in PP and Bakelite phases (a) and damaged area of bakelite (b) in PP/PMC/g-MA composite vs. strain for several imposed speed

More interesting, a higher average porosity is observed in the PP matrix as the fillers are added and as the velocity decreases (see Figure 12a). This is not in contradiction with the assessment that the average volumetric strain of composites increases with the imposed speed, as the volumetric strain in Bakelite follows an inverse trend (see Figure 12a). This is a consequence of this higher solicitation of bakelite as the tensile test speed increases. Beside these average variations, the histogram representing 
the frequencies of porosity levels in elements of the meshed structure reveals higher level of porosity for the speed $50 \mathrm{~mm} / \mathrm{min}$ (Figure 12b).

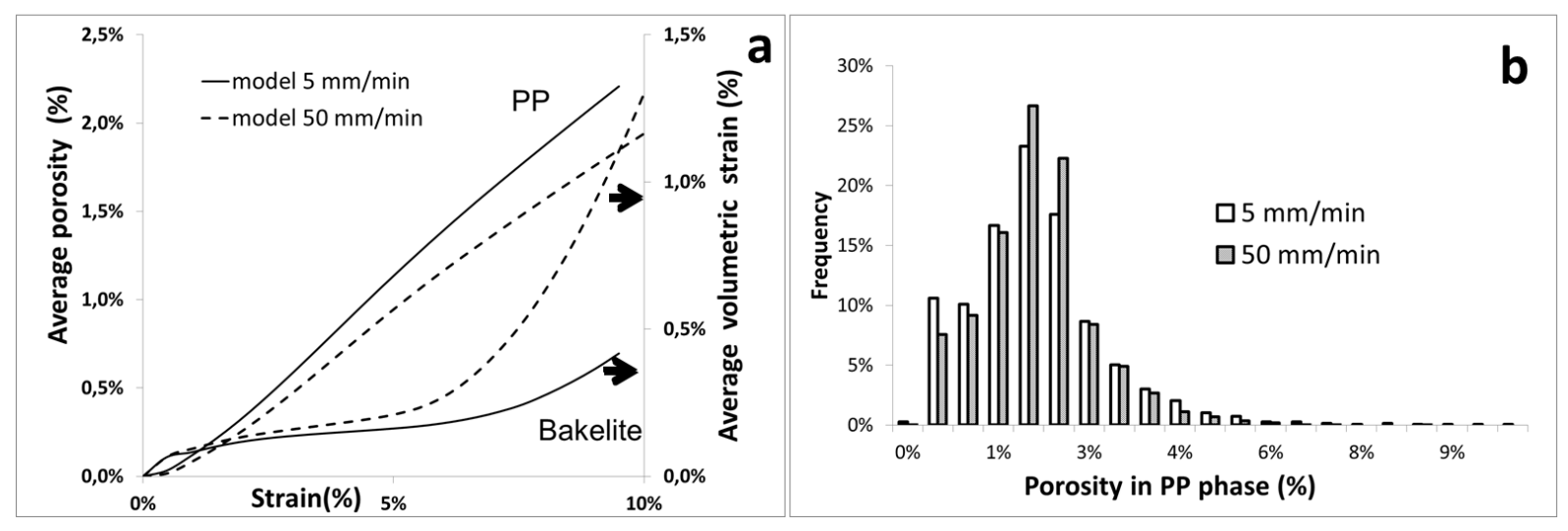

Figure 12: Average porosity of PP, average volumetric strain in Bakelite versus strain (a) and porosity repartition into PP phase

For these two-phase composites whose softer matrix is associated with viscoplastic behavior, the matrix accommodating capacity is annihilated at high solicitation rates. This leads to greater damage in particulate reinforcements when the interface is cohesive. The particulate reinforcement acts as a fuse, thus delaying the cavitation damage of the matrix.

\section{Conclusion}

The mechanical properties of a polypropylene based composites have been studied in this work. Instrumented (photomechanics) experiments (uniaxial tensile tests) were carried out on PP and PP filled with $20 \%$ by weight of phenolic molding compound waste powder to highlight the damage process. PP-g-MA was added as a compatibiliser. At first, global measured values (as volume variation and load during the test) lead, for the neat PP, to a set of mechanical parameters describing the GTN model that well reproduce the global stress/strain curve. Knowing the fillers mechanical properties, PP/PMC/g-MA is considered as a two phases composite as the adhesion between matrix and fillers is supposed to be perfect. A FE code is used both to predict composites behavior and to understand the damage micromechanisms involved during any during any structuraltest. It was found that bakelite can be a good candidate to reinforce polymer. As usual fillers, it improves Young's modulus and strength. Tensile tests performed with a higher strain rates 
velocities lead to both delay the cavitation damage of the matrix and increase the damage level of fillers. The charges concentrate then the damage limiting the cavitation which usually leads to a premature rupture of the composite.

[1] F. Bernardeau, D. Perrin, A.-S. Caro-Bretelle, J.-C. Benezet, and P. lenny, "Development of a recycling solution for waste thermoset material: waste source study, comminution scheme and filler characterization," J. Mater. Cycles Waste Manag., 2018.

[2] F. Bernardeau, D. Perrin, A. S. Caro, J. C. Benezet, and P. lenny, "Valorization of waste thermoset material as a filler in thermoplastic: Mechanical properties of phenolic molding compound waste-filled PP composites," J. Appl. Polym. Sci., vol. 135, no. 12, 2018.

[3] D. H. D Żuchowska, "Some physical properties of polypropylene-phenolic microsphere blends," Eur. Polym. J., vol. 27, pp. 355-357, 1991.

[4] W. Y. Chiang, W. C. Wu, and B. Pukánszky, "Modification of polypropylene, blending with resole type phenol-formaldehyde resins," Eur. Polym. J., vol. 30, no. 5, pp. 573-580, 1994.

[5] L. C. Y. Z. S. W. Y. Zhang, "Dynamically cured polypropylene/Novolac blends compatibilized with maleic anhydride- g- polypropylene," J. Appl. Polym. Sci., vol. 104, no. 5, pp. 3337-3346, 2007.

[6] A. L. Gurson, "Continuum Theory of Ductile Rupture by Void Nucleation and Growth: Part I-Yield Criteria and Flow Rules for Porous Ductile Media," J. Eng. Mater. Technol., vol. 99, no. 1, p. 2, 1977.

[7] V. Tvergaard, "On localization in ductile materials containing spherical voids," Int. J. Fract., vol. 18, no. 4, pp. 237-252, 1982.

[8] V. Tvergaard and A. Needleman, "Analysis of the cup-cone fracture in a round tensile bar," Acta Metall., vol. 32, no. 1, pp. 157-169, 1984.

[9] G. Boisot, L. Laiarinandrasana, J. Besson, C. Fond, and G. Hochstetter, "Experimental investigations and modeling of volume change induced by void growth in polyamide 11," Int. J. Solids Struct., vol. 48, no. 19, pp. 2642-2654, 2011.

[10] I. Sevostianov and M. Kachanov, "Effect of interphase layers on the overall elastic and conductive properties of matrix composites. Applications to 
nanosize inclusion," Int. J. Solids Struct., vol. 44, no. 3-4, pp. 1304-1315, 2007.

[11] N. Selles, N. Saintier, and L. Laiarinandrasana, "Voiding mechanisms in semicrystalline polyamide 6 during creep tests assessed by damage based constitutive relationships and finite elements calculations," Int. J. Plast., 2016.

[12] L. M. Kachanov and D. Krajcinovic, "Introduction to Continuum Damage Mechanics," Journal of Applied Mechanics, vol. 54. p. 481, 1987.

[13] A. Christmann, P. lenny, J. C. Quantin, A. S. Caro-Bretelle, and J. M. LopezCuesta, "Mechanical behaviour at large strain of polycarbonate nanocomposites during uniaxial tensile test," Polymer (Guildf)., vol. 52, no. 18, 2011.

[14] J. Lemaitre, "A Continuous Damage Mechanics Model for Ductile Fracture," J. Eng. Mater. Technol.(Trans. ASME), vol. 107, no. January 1985, pp. 83-89, 1985.

[15] G. Rousselier, "Ductile fracture models and their potential in local approach of fracture," Nucl. Eng. Des., vol. 105, no. 1, pp. 97-111, 1987.

[16] V. Tvergaard and A. Needleman, "Analysis of the Cup-Cone Round Tensile Fracture," Acta Metall., vol. 32, no. 1, pp. 157-169, 1984.

[17] V. Tvergaard, "Influence of voids on shear band instabilities under plane strain conditions," Int. J. Fract., vol. 17, no. 4, pp. 389-407, 1981.

[18] D. . Hughes, a Mahendrasingam, W. . Oatway, E. . Heeley, C. Martin, and W. Fuller, "A simultaneous SAXS/WAXS and stress-strain study of polyethylene deformation at high strain rates," Polymer (Guildf)., vol. 38, no. 26, pp. 64276430, 1997.

[19] F. Addiego, A. Dahoun, C. G'Sell, and J. M. Hiver, "Characterization of volume strain at large deformation under uniaxial tension in high-density polyethylene," Polymer (Guildf)., vol. 47, no. 12, pp. 4387-4399, 2006.

[20] A. Pawlak, "Cavitation during tensile deformation of high-density polyethylene," Polymer (Guildf)., vol. 48, no. 5, pp. 1397-1409, 2007.

[21] A. S. Ognedal, A. H. Clausen, T. Berstad, T. Seelig, and O. S. Hopperstad, "Void nucleation and growth in mineral-filled PVC - An experimental and numerical study," Int. J. Solids Struct., vol. 51, no. 7-8, pp. 1494-1506, 2014.

[22] S. Castagnet, S. Girault, J. L. Gacougnolle, and P. Dang, "Cavitation in strained polyvinylidene fluoride: Mechanical and X-ray experimental studies," 
Polymer (Guildf)., vol. 41, no. 20, pp. 7523-7530, 2000.

[23] J. Besson, R. Leriche, R. Foerch, and G. Cailletaud, "Object-Oriented Programming Applied to the Finite Element Method Part II. Application to Material Behaviors," Rev. Eur. des Éléments Finis, 2012. 